\title{
A Computational Approach to Verhulst-Pearl Model
}

\author{
J. Sunday ${ }^{1}$, A. A. James ${ }^{2}$, E. A. Ibijola ${ }^{3}$, R. B. Ogunrinde ${ }^{4}$, S. N. Ogunyebi ${ }^{5}$ \\ ${ }^{1}$ (Department of Mathematical Sciences, Adamawa State University, Mubi, Nigeria) \\ ${ }^{2}$ (Mathematics Division, School of Arts and Sciences, American University of Nigeria, Yola, Nigeria) \\ 3, 4,5(Department of Mathematical Sciences, Ekiti State University, Ado-Ekiti, Nigeria)
}

\begin{abstract}
This researchpaper examines a computational approach to solving the Verhulst-Pearl Model (often called the Logistic Model). The paper also examines the applications of such a model in some related fields. The aim of this research paper is to put forward the integrator derived by Odekunle, Adesanya and Sunday (2012B) as an alternative tool for solving Logistic Models. The results obtained from the integrator show that it is computationally reliable.
\end{abstract}

Keywords:Block Integrator, Computationally Reliable, IVPs, Models, Verhulst-Pearl Equation

AMS Subject Classification: 65L05, 65L06, 65D30

\section{Introduction}

The Verhulst-Pearl Model was published after Verhulst had read Thomas Malthus' "an essay on the principles of population". He derived his logistic model to describe the self-limiting growth of a biological population. Many animal species are fertile only for a brief period during the year and the young are born in a particular season so that by the time they are ready to eat solid food it will be plentiful. For this reason, the system might be better described by a discrete difference equation than a continuous differential equation. This is because not every existing animal will reproduce (a portion of them are male after all), not every female will be fertile, not every conception will be successful, and not every pregnancy will be successfully carried to term; the population increase will be some fraction of the present population. Therefore, if $y_{n}$ is the number of animals this year and $y_{n+1}$ is the number next year, then

$y_{n+1}=\alpha y_{n}$

Where $\alpha$ (the growth rate or fecundity) will approximate the evolution of the population. This model produces exponential growth without limit (Elert, 2007). Since every population is bound by physical limitations of its surrounding, some allowance must be made to restrict this growth. If there is a carryingcapacity of the environment, then the population may not exceed that capacity. If it does, the population would become extinct. This can be modeled by multiplying the population by a number that approaches zero as the population approaches its limit. If we normalize $y_{n}$ to this capacity, then the multiplier $\left(1-y_{n}\right)$ will suffice and the resulting logistic equation becomes,

$$
y_{n+1}=\alpha y_{n}\left(1-y_{n}\right)
$$

Alternatively, let $N$ represents population size and $t$ represents time, the logistic model is formalized by the differential equation,

$$
\frac{d N}{d t}=\alpha N\left(1-\frac{N}{k}\right)
$$

Where $\alpha$ remains the growth rate and $k$ the carrying capacity. From (3), the early unimpeded growth rate is modeled by the first term $+\alpha N$, latter as the population grows; the second term $\left(-\alpha N^{2} / k\right)$ which multiplied out becomes larger than the first as some members of the population $N$ interfere with each other by competing for some critical resources, such as food or living space. This antagonistic effect is called the bottleneck and is modeled by the value of the parameter $k$. This competition diminishes the combined growth rate, until the value of $N$ ceases to grow (this is called maturity of the population). Dividing both sides of (3) by $k$ gives,

$$
\frac{d N}{d t k}=\alpha \frac{N}{k}\left(1-\frac{N}{k}\right)
$$

Now setting $y=N / k$ gives the differential equation, 


$$
\frac{d y}{d t}=\alpha y(1-y)
$$

Substituting $\alpha=1$ in (5) and imposing an initial condition $y\left(t_{0}\right)=y_{0}$ leads to the special logistic initial value problem,

$y^{\prime}=y(1-y), y\left(t_{0}\right)=y_{o}$

It is important to note that (6) is of the form,

$y^{\prime}=f(x, y), y(a)=\eta \forall a \leq x \leq b$

Where $f$ is continuous within the interval of integration $[a, b]$. We assume that $f$ satisfies Lipchitz condition which guarantees the existence and uniqueness of solution of (7).

Definition 1 (Meyer, 1995)

A model is an object or concept that is used to represent something else. It is reality scaled down and converted to a form we can comprehend. Thus, a mathematical model is a model whose parts are mathematical concepts such as constants, variables, functions, inequalities, etc.

Therefore, the attempt to describe some part of the real - world in mathematical terms is called mathematical modeling. It is an endeavor as old as antiquity but as modern as tomorrow's newspaper. That is why the logistic (Verhulst-Pearl) model finds application in virtually all fields of human endeavour. Ogunwale et al. (2010), in their paper also stressed that logistic model is an important class of non-linear regression model with great applications in growth and population studies. Mathematical techniques now play an important role in planning, managerial decision-making and economics which have probably been the longest quantified of the social sciences (Burghes et al., 1980).

\section{Applications of the Logistic (Verhulst-Pearl) Models}

The Verhulst - Pearl Model finds applications in various fields, among which are;

1.2. Neural Networks: Logistic Models are often used in neural networks to introduce nonlinearity in the model and or to clamp signals within a specific range. A popular neural net element computes a linear combination of its input signals, and applies a bounded logistic function to the result; this model can be seen as a smoothed variant of the classical threshold neuron.

1.3. Statistics: logistic functions are used in several roles in statistics; firstly, they are the cumulative distribution function of the logistic family of distribution. Secondly, they are used in logistic regression to model how the probability $\mathrm{p}$ of an event may be affected by one or more explanatory variables, an example would be to have the model,

$$
p=P(a+b x)
$$

1.4. Medicine: another application of logistic equation is in medicine, where the logistic differential equation is used to model the growth of tumors. Let $X(t)$ be the size of the tumor at time $t$, then its dynamics are governed by;

$$
X^{\prime}=\alpha\left(1-\frac{X}{k}\right) X
$$

which is of the type,

$$
X^{\prime}=F(X) X, F^{\prime}(X) \leq 0
$$

where $F(X)$ is the proliferation rate of the tumor. If chemotherapy is started with a log - kill effect, the equation may be revised to be,

$$
X^{\prime}=\alpha\left(1-\frac{X}{k}\right) X-c(t) X
$$

where $c(t)$ is the therapy-induced death rate. In the idealized case of every long therapy, $c(t)$ can be modeled as a periodic function (of period T) or (in case of continuous infusion therapy) as a constant function, and one has that,

$$
\frac{1}{T} \int_{o}^{T} c(t) d t>\alpha \Rightarrow \lim _{t \rightarrow+\infty} x(t)=0
$$


i.e. if the average therapy - induced death rate is greater than the baseline proliferation rate, then there is the eradication of the disease, of course, this is an over - simplified model of both the growth and the therapy (e.g. it does not take into account the phenomenon of clonal resistance).

1.5. Chemistry: the concentration of reactants and products in autocatalytic reactions follows the logistic function.

1.6. Physics: it is applied in Fermi distribution in the sense that the logistic function determines the statistical distribution of fermions over the energy states of a system in thermal equilibrium. In particular, it is the distribution of the probabilities that each possible energy level is occupied by fermions, according to FermiDirac statistics.

1.7. Linguistics: in linguistic, the logistic function can be used to model language change, an innovation that was at first marginal but has now become more universally adopted.

1.8. Economics: the logistic function can be used to illustrate the progress of the diffusion of an innovation, infrastructures and energy source substitutions and the role of work in the economy as well as with the long economic cycle.

Block integrators for solving (7) have initially been proposed by Milne (1953) who used them as starting values for predictor-corrector algorithm, Rosser (1967) developed Milne's method in form of implicit integrators, and Shampine and Watts (1969) also contributed greatly to the development and application of block integrators. More recently, authors like Butcher (2003), Zarina et al. (2005), Awoyemi et al. (2007), Yahaya et al. (2010), Areo et al. (2011), Badmus et al. (2011), Ibijola et al. (2011), Chollom et al. (2012), Odekunle et al. (2012A) have all proposed LMMs to generate numerical solution to (7). These authors proposed integrators in which the approximate solution ranges from power series, Chebychev's, Lagrange's and Laguerre's polynomials. The advantages of LMMs over single step methods have been extensively discussed by Awoyemi (2001).

III. Construction of the Block Integrator(Odekunle, Adesanya and Sunday, 2012B)

Interpolation and collocation procedures were used by choosing interpolation point $s$ at a grid point and collocation points $r$ at all points giving rise to $\xi=s+r-1$ system of equations whose coefficients are determined by using appropriate procedures. The approximate solution to (7) is taken to be a combination of power series and exponential function given by,

$y(x)=\sum_{j=0}^{4} a_{j} x^{j}+a_{5} \sum_{j=0}^{5} \frac{\alpha^{j} x^{j}}{j !}$

with the first derivative given by,

$$
y^{\prime}(x)=\sum_{j=0}^{4} j a_{j} x^{j-1}+a_{5} \sum_{j=1}^{5} \frac{\alpha^{j} x^{j-1}}{(j-1) !}
$$

where $a_{j}, \alpha^{j} \in \square$ for $j=0(1) 5$ and $y(x)$ is continuously differentiable. Let the solution of (7) be sought on the partition $\pi_{N}: a=x_{0}<x_{1}<x_{2}<\ldots<x_{n}<x_{n+1}<\ldots<x_{N}=b$, of the integration interval $[a, b]$ with a constant step-size $h$, given by, $h=x_{n+1}-x_{n}, n=0,1, \ldots, N$.

Then, substituting (14) in (7) gives,

$$
f(x, y)=\sum_{j=0}^{4} j a_{j} x^{j-1}+a_{5} \sum_{j=1}^{5} \frac{\alpha^{j} x^{j-1}}{(j-1) !}
$$

Now, interpolating (3) at point $x_{n+s}, s=0$ and collocating (15) at points $x_{n+r}, r=0(1) 4$, leads to the following system of equations,

$$
A X=U
$$

where

$$
\begin{aligned}
& A=\left[a_{0}, a_{1}, a_{2}, a_{3}, a_{4}, a_{5}\right]^{T} \\
& U=\left[y_{n}, f_{n}, f_{n+1}, f_{n+2}, f_{n+3}, f_{n+4}\right]^{T} \\
& \text { and }
\end{aligned}
$$




$$
X=\left[\begin{array}{cccccc}
1 & x_{n} & x_{n}^{2} & x_{n}^{3} & x_{n}^{4} & \left(1+\alpha x_{n}+\frac{\alpha^{2} x_{n}^{2}}{2}+\frac{\alpha^{3} x_{n}^{3}}{6}+\frac{\alpha^{4} x_{n}^{4}}{24}+\frac{\alpha^{5} x_{n}^{5}}{120}\right) \\
0 & 1 & 2 x_{n} & 3 x_{n}^{2} & 4 x_{n}^{3} & \left(\alpha+\alpha^{2} x_{n}+\frac{\alpha^{3} x_{n}^{2}}{2}+\frac{\alpha^{4} x_{n}^{3}}{6}+\frac{\alpha^{5} x_{n}^{4}}{24}\right) \\
0 & 1 & 2 x_{n+1} & 3 x_{n+1}^{2} & 4 x_{n+1}^{3} & \left(\alpha+\alpha^{2} x_{n+1}+\frac{\alpha^{3} x_{n+1}^{2}}{2}+\frac{\alpha^{4} x_{n+1}^{3}}{6}+\frac{\alpha^{5} x_{n+1}^{4}}{24}\right) \\
0 & 1 & 2 x_{n+2} & 3 x_{n+2}^{2} & 4 x_{n+2}^{3} & \left(\alpha+\alpha^{2} x_{n+2}+\frac{\alpha^{3} x_{n+2}^{2}}{2}+\frac{\alpha^{4} x_{n+2}^{3}}{6}+\frac{\alpha^{5} x_{n+2}^{4}}{24}\right) \\
0 & 1 & 2 x_{n+3} & 3 x_{n+3}^{2} & 4 x_{n+3}^{3} & \left(\alpha+\alpha^{2} x_{n+3}+\frac{\alpha^{3} x_{n+3}^{2}}{2}+\frac{\alpha^{4} x_{n+3}^{3}}{6}+\frac{\alpha^{5} x_{n+3}^{4}}{24}\right) \\
0 & 1 & 2 x_{n+4} & 3 x_{n+4}^{2} & 4 x_{n+4}^{3} & \left(\alpha+\alpha^{2} x_{n+4}+\frac{\alpha^{3} x_{n+4}^{2}}{2}+\frac{\alpha^{4} x_{n+4}^{3}}{6}+\frac{\alpha^{5} x_{n+4}^{4}}{24}\right)
\end{array}\right]
$$

Solving (16), for $a_{j}{ }^{\prime} s, j=0(1) 5$ and substituting back into (13) gives a continuous linear multistep method of the form,

$$
y(x)=\alpha_{0}(x) y_{n}+h \sum_{j=0}^{4} \beta_{j}(x) f_{n+j}
$$

where $\alpha_{0}=1$ and the coefficients of $f_{n+j}$ gives

$$
\left.\begin{array}{l}
\beta_{0}=\frac{1}{720}\left(6 t^{5}-75 t^{4}+350 t^{3}-750 t^{2}+720 t\right) \\
\beta_{1}=-\frac{1}{360}\left(12 t^{5}-135 t^{4}+520 t^{3}-720 t^{2}\right) \\
\beta_{2}=\frac{1}{60}\left(3 t^{5}-30 t^{4}+95 t^{3}-90 t^{2}\right) \\
\beta_{3}=-\frac{1}{360}\left(12 t^{5}-105 t^{4}+280 t^{3}-240 t^{2}\right) \\
\beta_{4}=\frac{1}{720}\left(6 t^{5}-45 t^{4}+110 t^{3}-90 t^{2}\right)
\end{array}\right\}
$$

where $t=\left(x-x_{n}\right) / h$. Evaluating (17) at $t=1(1) 4$ gives a continuous discrete block scheme of the form,

$$
A^{(0)} \mathbf{Y}_{m}=\mathbf{E} \mathbf{y}_{n}+h d \mathbf{f}\left(\mathbf{y}_{n}\right)+h b \mathbf{F}\left(\mathbf{Y}_{m}\right)
$$

where

$$
\begin{aligned}
& \mathbf{Y}_{m}=\left[y_{n+1}, y_{n+2}, y_{n+3}, y_{n+4}\right]^{T}, \mathbf{y}_{n}=\left[y_{n-3}, y_{n-2}, y_{n-1}, y_{n}\right]^{T}, \\
& \mathbf{F}\left(\mathbf{Y}_{m}\right)=\left[f_{n+1}, f_{n+2}, f_{n+3}, f_{n+4}\right]^{T}, \mathbf{f}\left(\mathbf{y}_{n}\right)=\left[f_{n-3}, f_{n-2}, f_{n-1}, f_{n}\right]^{T},
\end{aligned}
$$$$
A^{(0)}=\left[\begin{array}{llll}
1 & 0 & 0 & 0 \\
0 & 1 & 0 & 0 \\
0 & 0 & 1 & 0 \\
0 & 0 & 0 & 1
\end{array}\right], \mathbf{E}=\left[\begin{array}{llll}
0 & 0 & 0 & 1 \\
0 & 0 & 0 & 1 \\
0 & 0 & 0 & 1 \\
0 & 0 & 0 & 1
\end{array}\right], \quad d=\left[\begin{array}{llll}
0 & 0 & 0 & \frac{251}{720} \\
0 & 0 & 0 & \frac{29}{90} \\
0 & 0 & 0 & \frac{27}{80} \\
0 & 0 & 0 & \frac{14}{45}
\end{array}\right]
$$

$$
b=\left[\begin{array}{cccc}
\frac{323}{360} & \frac{-11}{30} & \frac{53}{360} & \frac{-19}{720} \\
\frac{62}{45} & \frac{4}{15} & \frac{2}{45} & \frac{-1}{90} \\
\frac{51}{40} & \frac{9}{10} & \frac{21}{40} & \frac{-3}{80} \\
\frac{64}{45} & \frac{8}{15} & \frac{64}{45} & \frac{14}{45}
\end{array}\right]
$$




\subsection{Order of the New Block Integrator \\ IV. Analysis of Basic Properties of the Block Integrator}

Let the linear operator $L\{y(x) ; h\}$ associated with the block (19) be defined as,

$$
L\{y(x) ; h\}=A^{(0)} Y_{m}-E y_{n}-h d f\left(y_{n}\right)-h b F\left(Y_{m}\right)
$$

Expanding (20) using Taylor series and comparing the coefficients of $h$ gives,

$$
L\{y(x) ; h\}=c_{0} y(x)+c_{1} h y^{\prime}(x)+c_{2} h^{2} y^{\prime \prime}(x)+\ldots+c_{p} h^{p} y^{p}(x)+c_{p+1} h^{p+1} y^{p+1}(x)+\ldots
$$

\section{Definition 2}

The linear operator $L$ and the associated continuous linear multistep method (17) are said to be of order $p$ if $c_{0}=c_{1}=c_{2}=\ldots=c_{p}$ and $c_{p+1} \neq 0 . c_{p+1}$ is called the error constant and the local truncation error is given by,

$$
t_{n+k}=c_{p+1} h^{(p+1)} y^{(p+1)}\left(x_{n}\right)+O\left(h^{p+2}\right)
$$

For our method,

$$
L\{y(x) ; h\}=\left[\begin{array}{llll}
1 & 0 & 0 & 0 \\
0 & 1 & 0 & 0 \\
0 & 0 & 1 & 0 \\
0 & 0 & 0 & 1
\end{array}\right]\left[\begin{array}{l}
y_{n+1} \\
y_{n+2} \\
y_{n+3} \\
y_{n+4}
\end{array}\right]-\left[\begin{array}{l}
1 \\
1 \\
1 \\
1
\end{array}\right]\left[y_{n}\right]-h\left[\begin{array}{ccccc}
\frac{251}{720} & \frac{323}{360} & \frac{-11}{30} & \frac{53}{360} & \frac{-19}{720} \\
\frac{29}{90} & \frac{62}{45} & \frac{4}{15} & \frac{2}{45} & \frac{-1}{90} \\
\frac{27}{80} & \frac{51}{40} & \frac{9}{10} & \frac{21}{40} & \frac{-3}{80} \\
\frac{14}{45} & \frac{64}{45} & \frac{8}{15} & \frac{64}{45} & \frac{14}{45}
\end{array}\right]\left[\begin{array}{l}
f_{n} \\
f_{n+1} \\
f_{n+2} \\
f_{n+3} \\
f_{n+4}
\end{array}\right]=\mathrm{O}
$$

Expanding (23) in Taylor series gives,

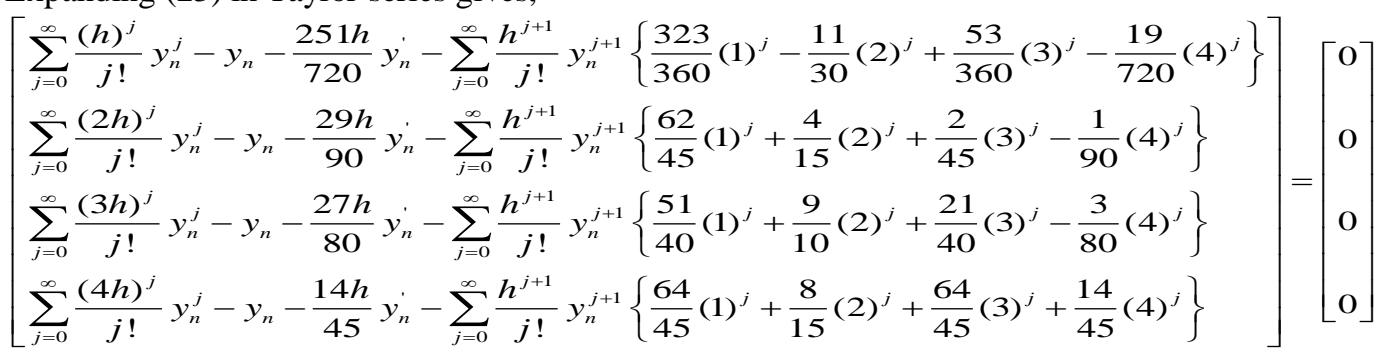

Hence, $c_{0}=c_{1}=c_{2}=c_{3}=c_{4}=c_{5}=0, c_{6}=[1.88(-02), 1.11(-02), 1.88(-02),-8.47(-03)]^{T}$. Therefore, the block integrator is of order five.

\subsection{Zero Stability}

\section{Definition 3}

The block integrator (19) is said to be zero-stable, if the roots $z_{s}, s=1,2, \ldots, k$ of the first characteristic polynomial $\rho(z)$ defined by $\rho(z)=\operatorname{det}\left(z \mathbf{A}^{(0)}-\mathbf{E}\right)$ satisfies $\left|z_{s}\right| \leq 1$ and every root satisfying $\left|z_{s}\right| \leq 1$ have multiplicity not exceeding the order of the differential equation. Moreover, as $h \rightarrow 0$, $\rho(z)=z^{r-\mu}(z-1)^{\mu}$ where $\mu$ is the order of the differential equation, $r$ is the order of the matrices $\mathbf{A}^{(0)}$ and $\mathbf{E}$ (see Awoyemiet al. (2007) for details).

For our new integrator,

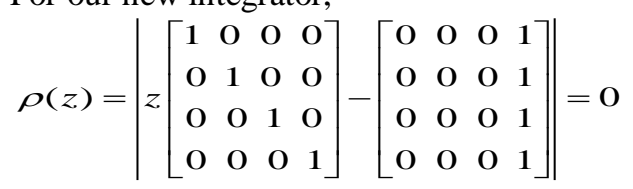

$\rho(z)=z^{3}(z-1)=0, \Rightarrow z_{1}=z_{2}=z_{3}=0, z_{4}=1$. Hence, the block integrator is zero-stable. 


\subsection{Consistency}

The block integrator is consistent since it has order $p=5 \geq 1$.

\subsection{Convergence}

The new block integrator is convergent by consequence of Dahlquist theorem below.

Theorem 1 (Dahlquist, 1956)

The necessary and sufficient conditions that a continuous LMM be convergent are that it be consistent and zerostable.

\subsection{Region of Absolute Stability}

\section{Definition 4}

The method (19) is said to be absolutely stable if for a given $h$, all the roots $z_{s}$ of the characteristic polynomial $\pi(z, \bar{h})=\rho(z)+\bar{h} \sigma(z)=0$ satisfies $z_{s}<1, s=1,2, \ldots, n$ where $\bar{h}=\lambda h$ and $\lambda=\frac{\partial f}{\partial y}$.

We shall adopt the boundary locus method for the region of absolute stability of the block method. Substituting the test equation $y^{\prime}=-\lambda y$ into the block formula gives,

$\mathbf{A}^{(0)} \mathbf{Y}_{m}(r)=\mathbf{E y}_{n}(r)-h \lambda \mathbf{D} y_{n}(r)-h \lambda \mathbf{B Y} \mathbf{Y}_{m}(r)$

Thus,

$\bar{h}(r, h)=-\left(\frac{\mathbf{A}^{(0)} Y_{m}(r)-\mathbf{E} y_{n}(r)}{\mathbf{D} y_{n}(r)+\mathbf{B} Y_{m}(r)}\right)$

Writing (27) in trigonometric ratios gives,

$\bar{h}(\theta, h)=-\left(\frac{\mathbf{A}^{(0)} Y_{m}(\theta)-\mathbf{E} y_{n}(\theta)}{\mathbf{D} y_{n}(\theta)+\mathbf{B} Y_{m}(\theta)}\right)$

where $r=e^{i \theta}$. Equation (28) is our characteristic/stability polynomial. Applying (28) to our method, we have,

$\bar{h}(\theta, h)=\frac{(\cos 2 \theta)(\cos 3 \theta)(\cos \theta)-(\cos 2 \theta)(\cos 3 \theta)(\cos 4 \theta)(\cos \theta)}{\frac{1}{5}(\cos 2 \theta)(\cos 3 \theta)(\cos \theta)-\frac{1}{5}(\cos 2 \theta)(\cos 3 \theta)(\cos 4 \theta)(\cos \theta)}$

which gives the stability region shown in fig. 1 below.

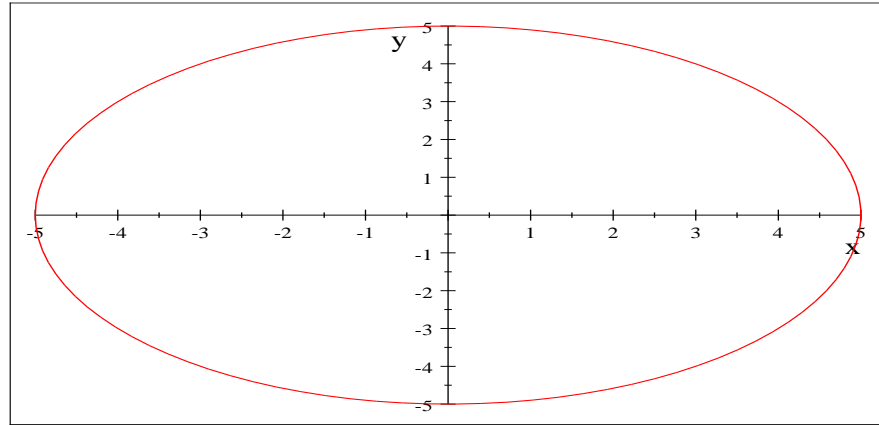

Fig. 1: Region of Absolute Stability of the Block Integrator

\section{Numerical Implementation}

We shall now proceed to implement the block integrator (19) on a special case of logistic (Verhulst Pearl) Model. The implementation is carried out using Matlab application language.

\section{Problem 1}

Consider the Verhulst- Pearl (Logistic) Model,

$y^{\prime}=y(1-y), \quad y(0)=0.5$

with the theoretical solution,

$y(t)=\frac{0.5}{\left(0.5+0.5 e^{-t}\right)}$ 
The algorithm for implementing the block integrator (19) on (30) using matlab is given by,

function output $=f(x, y)$

output $=$ ?;

functionexactsol $=f r(x)$

exact sol=?;

$x 0=? ; y 0=? ; h=? ;$

disp('x-value Exact Solution Computed Solution Error')

for $j=1: 1: 4$;

$i=1: 4$

$f \mathrm{O}=g(x \mathrm{O}, y \mathrm{O})$

$x(i)=x \mathrm{O}+i * h$;

$y(i)=y 0+(i * h)^{*} f 0+\left(\left((i * h)^{\wedge} 2\right) / 2\right) * d x(x 0, y 0)$

$+\left(\left((i * h)^{\wedge} 3\right) / 6\right) * d d x(x 0, y 0)+\ldots$

$y i(x)=g i_{L}$

$m i=$ toc;

erri $=\operatorname{abs}(f r(x(i))-y r i)$;

fprint $f(\% 2.4 f \% 3.16 f \% 3.16 f \% 1.6 e \% 2.4 f$

$\backslash n, x(i), f r(x(i)), y r i$, erri, $m i)$

$x \mathrm{O}=x(4) ; y \mathrm{O}=y r(4)$;

end

Note that: $y(x)=g_{L}$, for $g_{L}=\alpha_{0}(x) y_{n}+h \beta_{j}(x) f_{n+j}, j=0(1) 4$

On the application of (19) on (30) we obtain the following numerical results at different values of time $t$. We shall use the following notation in the tables below;

$\boldsymbol{E R R}$ - |Exact Solution-Computed Result|

Table 1: Performance of the Block Integrator (19) on Problem (1) with $h=0.1$

\begin{tabular}{cccc}
\hline & $t$ Exact solution & Computed solution & ERR \\
\hline 0.10000 .5249791874789400 & 0.5249791908697810 & $3.390841 \mathrm{e}-009$ \\
0.2000 & 0.5498339973124780 & 0.5498339991814910 & $1.869013 \mathrm{e}-009$ \\
0.3000 & 0.5744425168116590 & 0.5744425201061023 & $3.294443 \mathrm{e}-009$ \\
0.4000 & 0.5986876601124520 & 0.5986876562777278 & $3.834724 \mathrm{e}-009$ \\
0.5000 & 0.6224593312018546 & 0.6224593350408475 & $3.838993 \mathrm{e}-009$ \\
0.6000 & 0.6456563062257954 & 0.6456563070425375 & $8.167421 \mathrm{e}-010$ \\
0.7000 & 0.6681877721681662 & 0.6681877759967531 & $3.828587 \mathrm{e}-009$ \\
0.8000 & 0.6899744811276125 & 0.6899744751554371 & $5.972175 \mathrm{e}-009$ \\
0.9000 & 0.7109495026250039 & 0.7109495023747208 & $2.502831 \mathrm{e}-010$ \\
1.0000 & 0.7310585786300049 & 0.7310585764813252 & $2.148680 \mathrm{e}-009$
\end{tabular}

Table 2: Performance of the Block Integrator (19) on Problem (1) with $h=0.01$

\begin{tabular}{cccc}
\hline & $t$ Exact solution & Computed solution & ERR \\
\hline 0.0100 & 0.5024999791668749 & 0.5024999791668754 & $4.440892 \mathrm{e}-016$ \\
0.0200 & 0.5049998333399998 & 0.5049998333399999 & $1.110223 \mathrm{e}-016$ \\
0.0300 & 0.5074994375506203 & 0.5074994375506208 & $4.440892 \mathrm{e}-016$ \\
0.0400 & 0.5099986668799655 & 0.5099986668799654 & $1.110223 \mathrm{e}-016$ \\
0.0500 & 0.5124973964842103 & 0.5124973964842114 & $1.110223 \mathrm{e}-015$ \\
0.0600 & 0.5149955016194100 & 0.5149955016194107 & $6.661338 \mathrm{e}-016$ \\
0.0700 & 0.5174928576663898 & 0.5174928576663909 & $1.110223 \mathrm{e}-015$ \\
0.0800 & 0.5199893401555819 & 0.5199893401555816 & $2.220446 \mathrm{e}-016$ \\
0.0900 & 0.5224848247918001 & 0.5224848247918019 & $1.776357 \mathrm{e}-015$ \\
0.1000 & 0.5249791874789400 & 0.5249791874789409 & $8.881784 \mathrm{e}-016$
\end{tabular}

\section{Conclusion}

We conclude that the block integrator in (19) is computationally reliable and we therefore recommend it as an alternative approach for estimating the solution of Verhulst-Pearl Models and other related models that 
occur in the form of equation (7). We also observed that the integrator is zero-stable, consistent and convergent. Thus, satisfying the basic properties of the block integrator.

\section{Authors' Profile}

J. Sunday: received his M.Sc. in Mathematics (Numerical and Computational Mathematics) in 2011 from the University of Ado-Ekiti (now Ekiti State University), Nigeria. A member of the Mathematical Association of Nigeria (MAN), Nigeria Mathematical Society (NMS) and an associate member of the Institute of Strategic Management Nigeria (ISMN). A lecturer with the Department of Mathematical Sciences, Adamawa State University, Mubi, Nigeria. Currently on a Ph.D programme at the Modibbo Adama University of Technology, Yola. His major research interests are in numerical analysis.

A.A James: received his M.Sc. in Mathematics (Complex Analysis) in 2008 from the University of Ilorin, also owns post graduate degrees in education and computer from UsmanDanfodia University Sokoto and University of Ilorin respectively. A member of the Mathematical Association of Nigeria (MAN), Nigeria Mathematical Society (NMS) and IEEE.A faculty member with the Mathematics Division of the American University of Nigeria, Yola, Nigeria. Currently on a Ph.D programme at the Modibbo Adama University of Technology, Yola. His major research interest is in numerical methods to solving IVP ODE problems.

Prof. E. A. Ibijola: is a professor of Numerical Analysis. He received his Ph.D from the University of Benin, Nigeria in 1997. He has published over 130 research papers, $98 \%$ of which are international research papers. He is a member of many professional bodies. He has graduated many Ph.D, M.Sc. and Undergraduate students. He has also taught in many universities outside Nigeria. He is now the head of the Department of Mathematical Sciences, University of Ado-Ekiti, Nigeria.

Dr. (Mrs.) R. B. Ogunrinde: received her Ph.D in Mathematics from the University of Ado-Ekiti (now Ekiti State University), Nigeria in 2010. She has published so many research papers. She is the postgraduate coordinator of the Department of Mathematical Sciences, Ekiti State University, Nigeria where she works as a lecturer. She is a member of many professional bodies. Her research interests are in numerical analysis.

S. N. Ogunyebi: received his M.Tech. in Mathematics from the Federal University of Technology, Akure. He has published so many research papers. He is a lecturer with the Department of Mathematical Sciences, Ekiti State University, Nigeria. He is a member of many professional bodies. Currently on his Ph.D programme at the Federal University of Technology, Akure, Nigeria.

\section{References}

[1] D. N. Burghesand A. D. Wood, "Mathematical models in the Social, Management and Life Sciences", Ellis Harwood Limited, 1980.

[2] G. Elert, "Measuring Chaos", The Chaos Hypertext book, 2007.

[3] W. J. Meyer, "Concepts of Mathematical Modeling", Mc Grow-Hill Book Company, 1995.

[4] R. E. Mickens, "Nonstandard Finite Difference Models of Differential Equations", World Scientific, Singapore, 1994.

[5] O. D. Ogunwale, O. Y. Halid, and J. Sunday, (2010), "On an Alternative Method of Estimation of

[6] PolynomialRegression Models”, Australian Journal of Basic and Applied Sciences, vol. 4, no. 8, pp. 3585-3590, 2010.

[7] E. A. Areo, R. A. Ademiluyi and P. O. Babatola, "Three Steps Hybrid LinearMultistep Method for the Solution of First-Order Initial Value Problems in Ordinary Differential Equations",Journal of Mathematical Physics,vol. 19, pp. 261-266, 2011.

[8] D. O. Awoyemi, R. A. Ademiluyi and W. Amuseghan, "Off-grids Exploitation in the Development of More Accurate Method for the Solution of ODEs", Journal of Mathematical Physics, vol. 12, pp. 379-386, 2011.

[9] A.M. Badmus, D. W. Mishelia, "Some Uniform Order Block Method for the Solution of First-Order Ordinary Differential Equations", Journal of Nigerian Association of Mathematical Physics, vol. 19, pp. 149-154, 2011.

[10] J. C. Butcher, "Numerical Methods for Ordinary Differential Equations",West Sussex: John Wiley \& Sons Ltd, 2003.

[11] J. P. Chollom, I. O. Olatunbosun, andS. Omagu, "A Class of A-Stable Block Explicit Methods for the Solution of Ordinary Differential Equations", Research Journal of Mathematics and Statistics, vol. 4, no. 2,pp. 52-56, 2012.

[12] G. G. Dahlquist, "Convergence and Stability in the Numerical Integration of Ordinary Differential Equations", Math. Scand.,vol. 4, pp. 33-50, 1956.

[13] E. A. Ibijola, Y. Skwame and G. Kumleng, "Formulation of Hybrid Method of Higher Step-sizes Through the Continuous Multistep Collocation”,American Journal of Scientific and Industrial Research, vol. 2, no. 2, pp. 161-173, 2011.

[14] W. E. Milne, "Numerical Solution of Differential Equations", New York, Willey, 1953.

[15] M. R. Odekunle, A. O. Adesanya, and J. Sunday, "A New Block Integrator for the Solution of Initial Value Problems of FirstOrder Ordinary Differential Equations”, International Journal of Pure and Applied Science and Technology, vol. 11, no. 1,pp. 92$100,2012 \mathrm{~A}$.

[16] M. R. Odekunle, A. O. Adesanya, and J. Sunday, "4-Point Block Method for the Direct Integration of First-Order Ordinary Differential Equations", International Journal of Engineering Research and Applications, vol. 2, no. 5,pp. 1182-1187, 2012B.

[17] J. B. Rosser,"A Runge-Kutta Method for all Seasons”, SIAM Review, vol. 9, pp. 417-452, 1967.

[18] L. F. Shampine and H. A. Watts, "Block Implicit One-Step Methods",Mathematics of Computation, vol. 23, no. 108,pp. 731-740, 1969.

[19] Y. A. Yahaya andU. Mohammed, "Fully Implicit Three Point Block Backward Differentiation Formulae for Solution of FirstOrder IVPs",Leonardo Journal of Sciences,vol. 16, no. 1,pp. 21-30, 2010.

[20] B. I. Zarina, S. Mohammed, I. Kharil, and M. Zanariah, "Block Method for Generalized Multistep Adams Method and Backward Differentiation Formula in Solving First-Order ODEs", Mathematika, pp. 25-33, 2005. 